\title{
New Luminescent Solids in the Ln-W(Mo)-Te-O-(Cl) Systems
}

\section{Supporting Information}

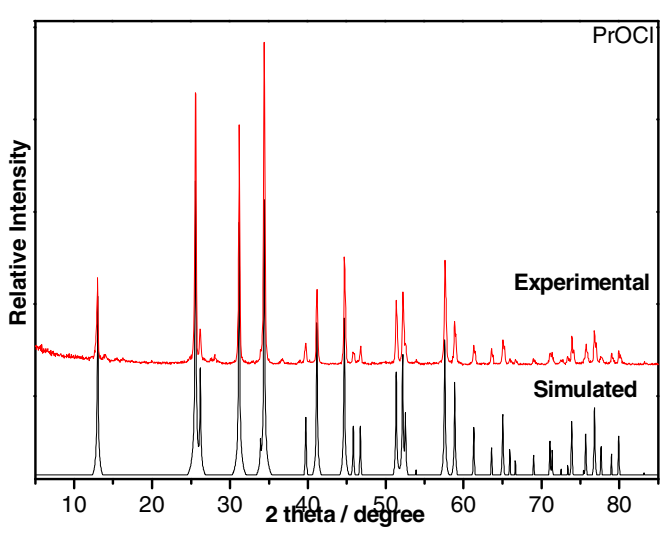

(a)

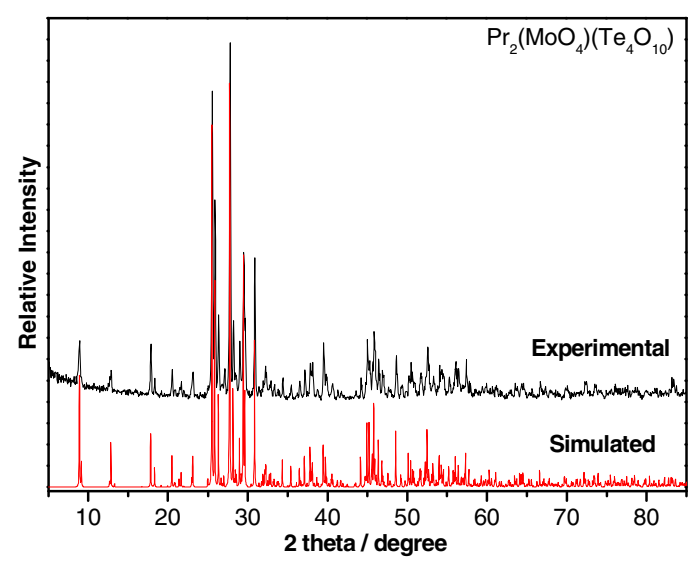

(c)

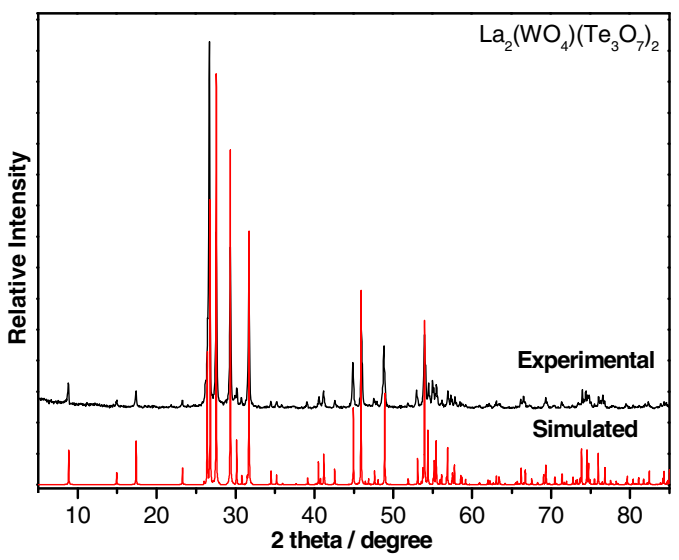

(e)

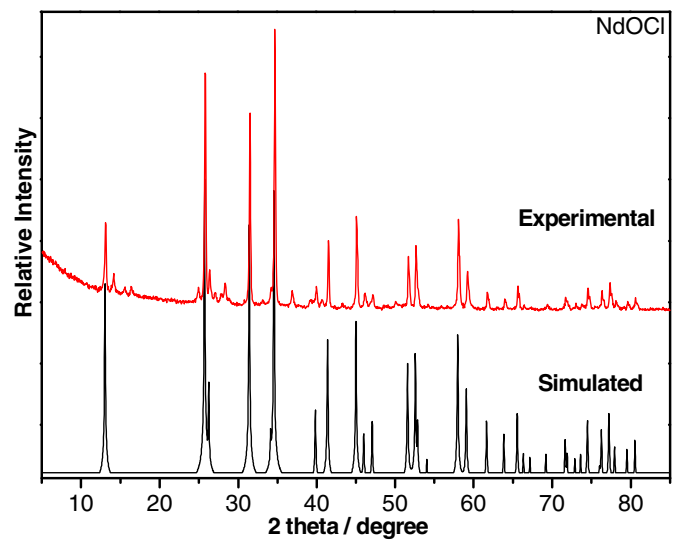

(b)

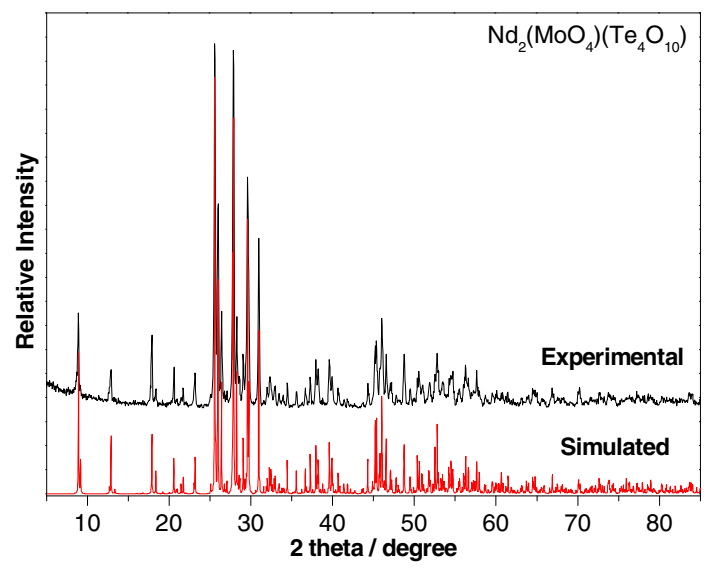

(d)

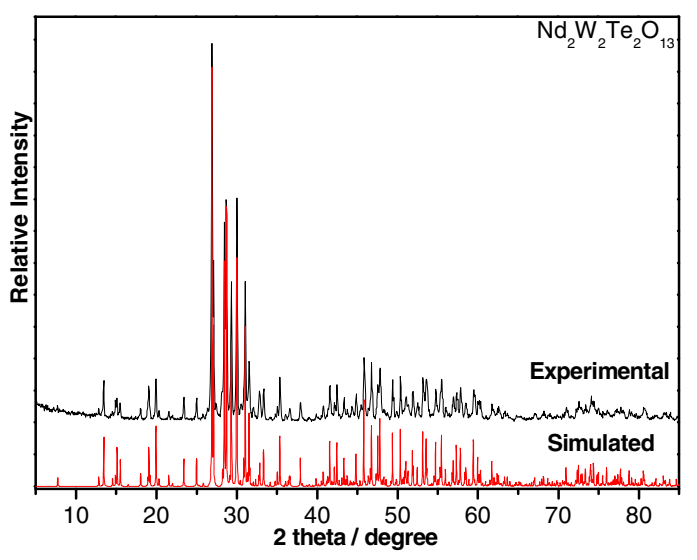

(f) 


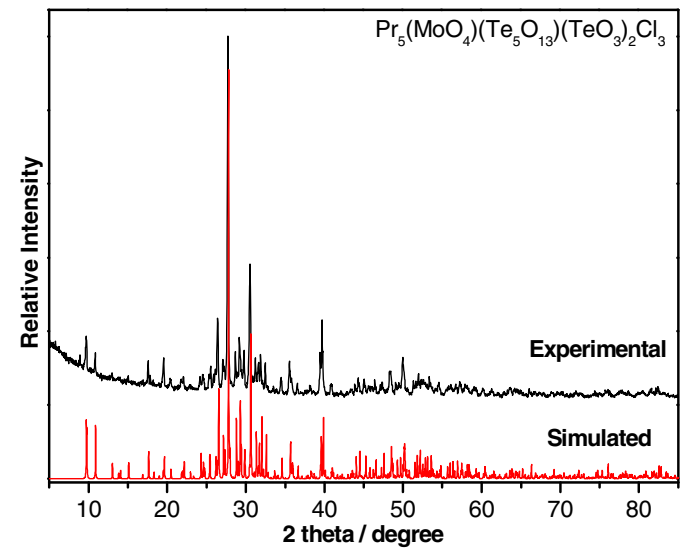

(g)

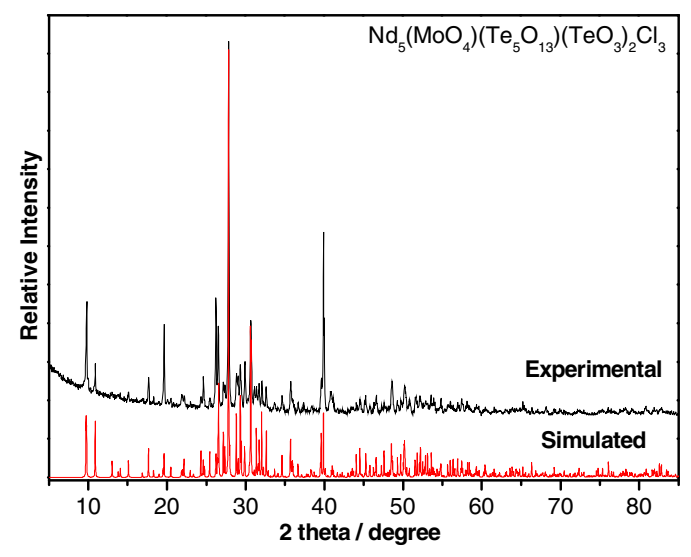

(i)

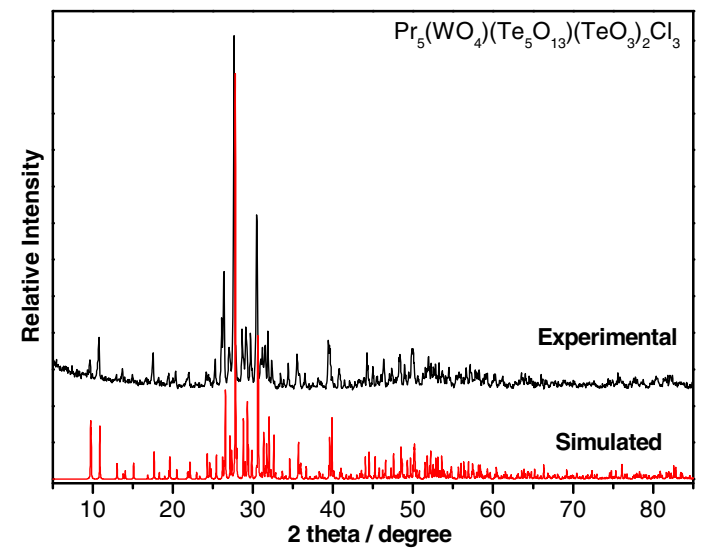

(h)

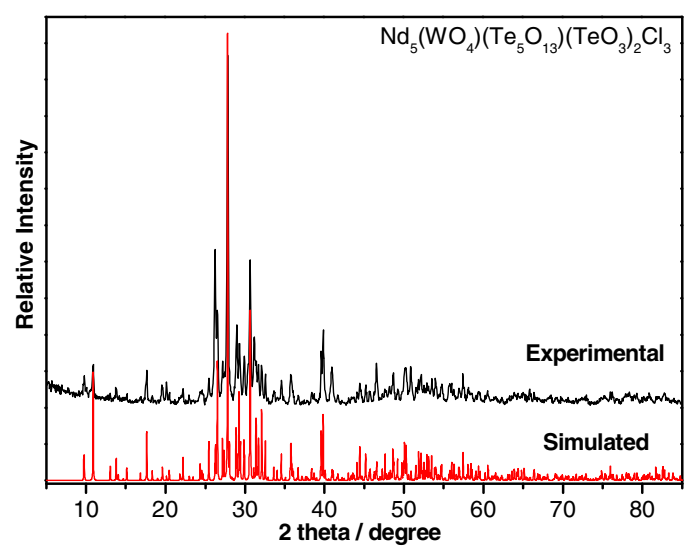

(j)

Figure S1. Simulated and experimental XRD powder patterns for $\mathrm{LnOCl}(\mathrm{Ln}=\mathrm{Pr}, \mathrm{Nd})$, $\mathrm{Ln}_{2}\left(\mathrm{MoO}_{4}\right)\left(\mathrm{Te}_{4} \mathrm{O}_{10}\right) \quad(\mathrm{Ln}=\mathrm{Pr}, \quad \mathrm{Nd}), \quad \mathrm{La}_{2}\left(\mathrm{WO}_{4}\right)\left(\mathrm{Te}_{3} \mathrm{O}_{7}\right)_{2}, \quad \mathrm{Nd}_{2} \mathrm{~W}_{2} \mathrm{Te}_{2} \mathrm{O}_{13}$ and $\mathrm{Ln}_{5}\left(\mathrm{MO}_{4}\right)\left(\mathrm{Te}_{5} \mathrm{O}_{13}\right)\left(\mathrm{TeO}_{3}\right)_{2} \mathrm{Cl}_{3}(\mathrm{Ln}=\mathrm{Pr}, \mathrm{Nd} ; \mathrm{M}=\mathrm{Mo}, \mathrm{W})$.

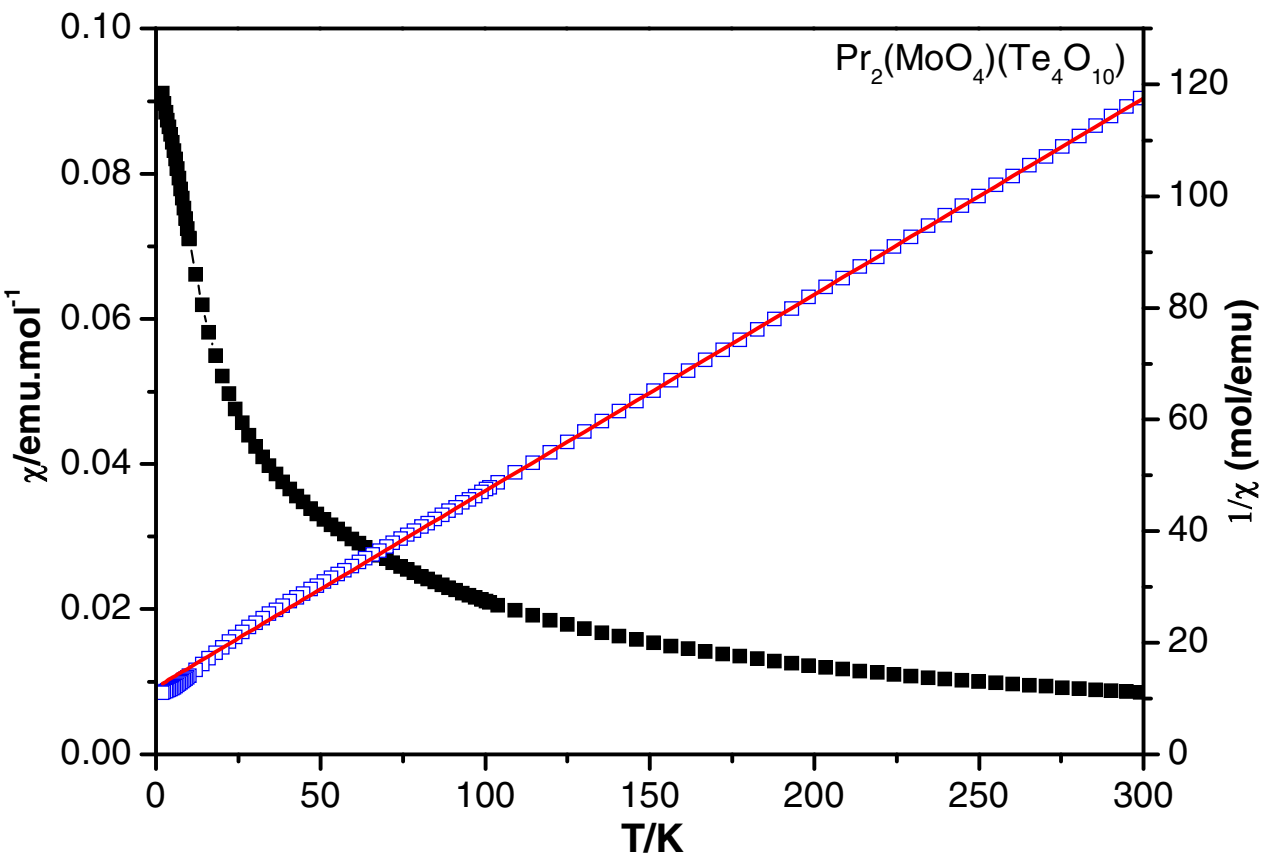

(a) 


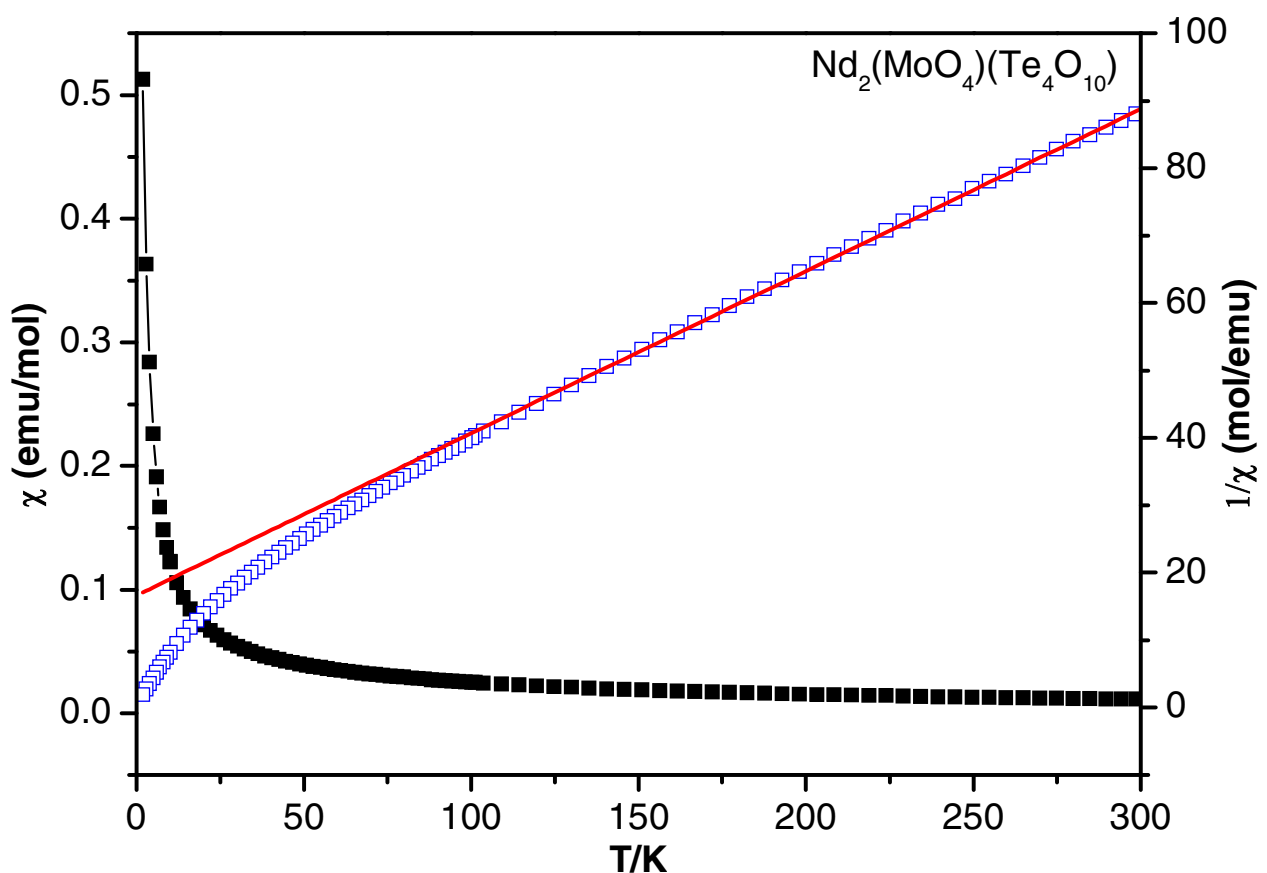

(b)

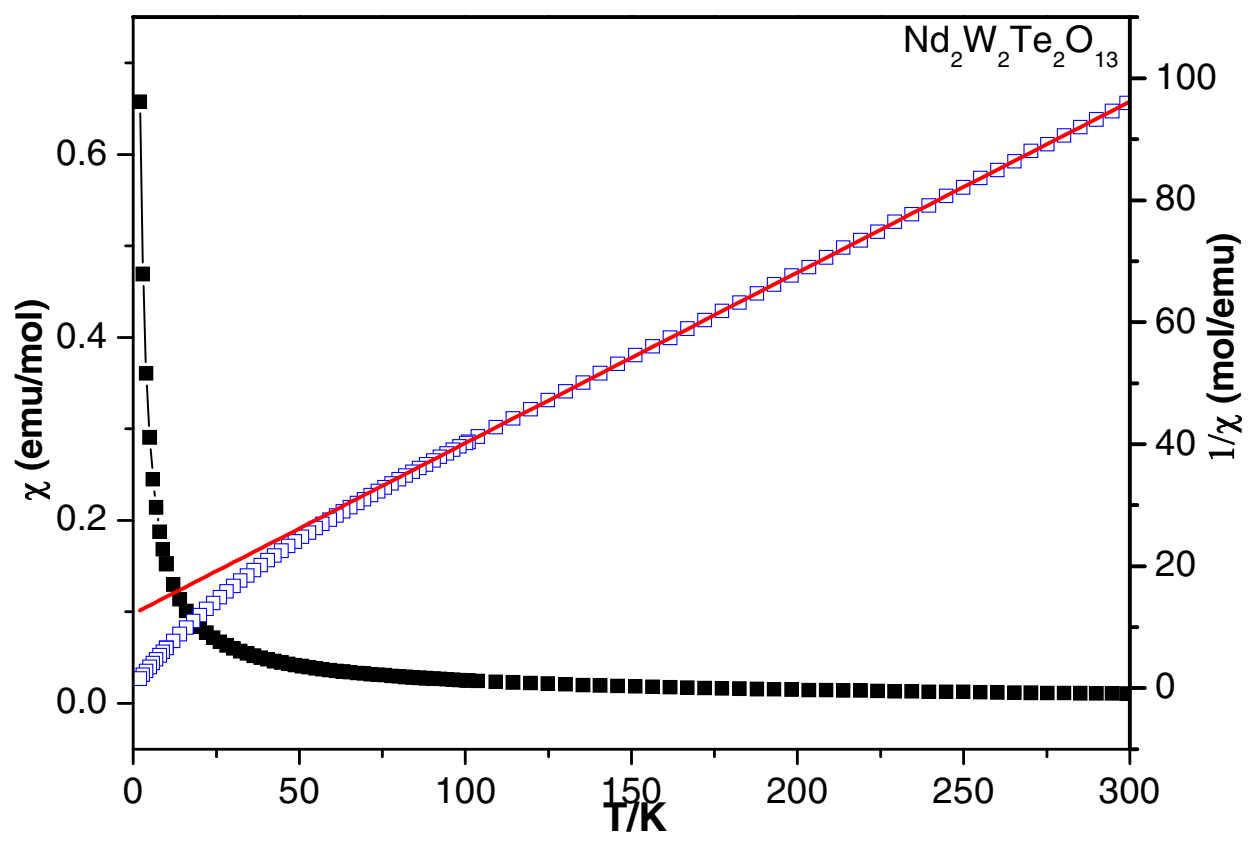

(c) 


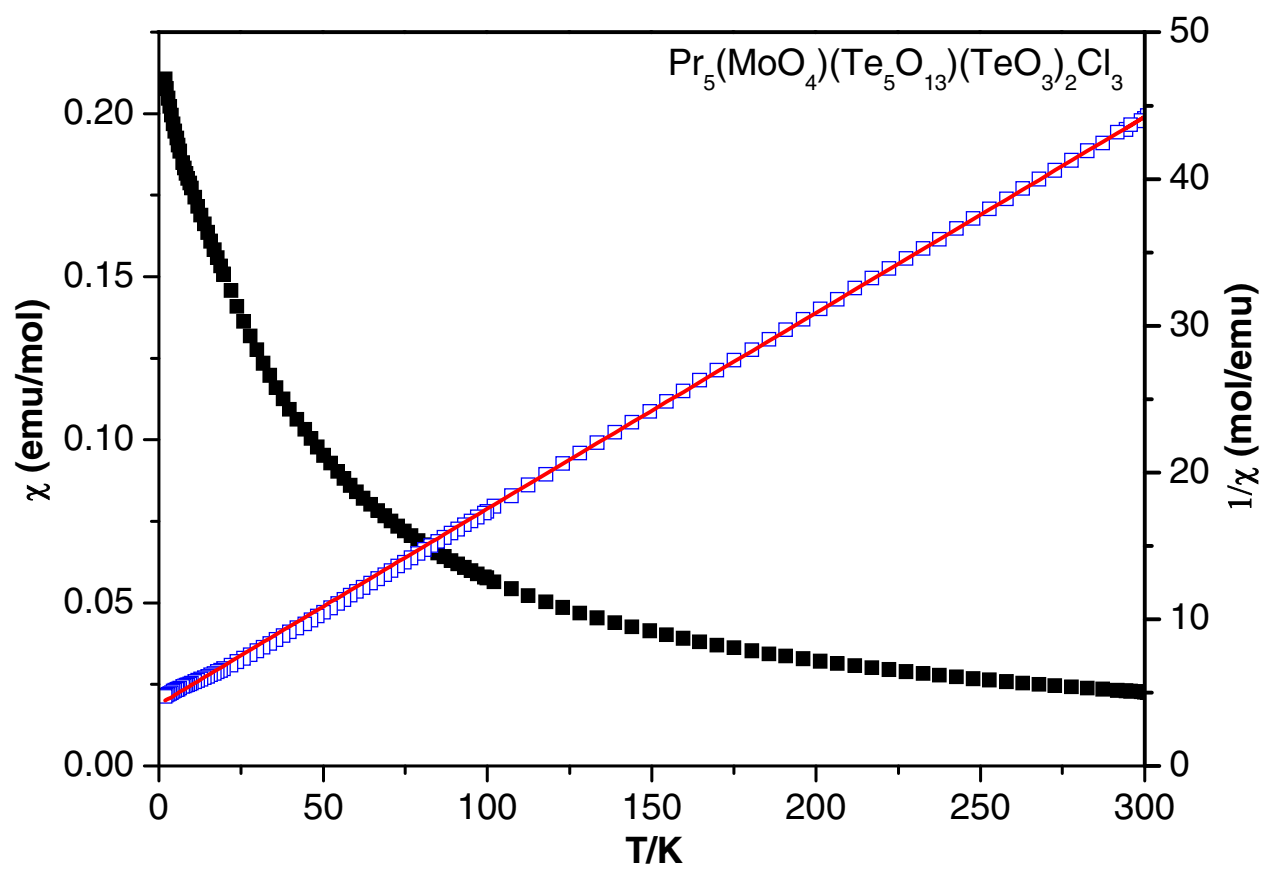

(d)

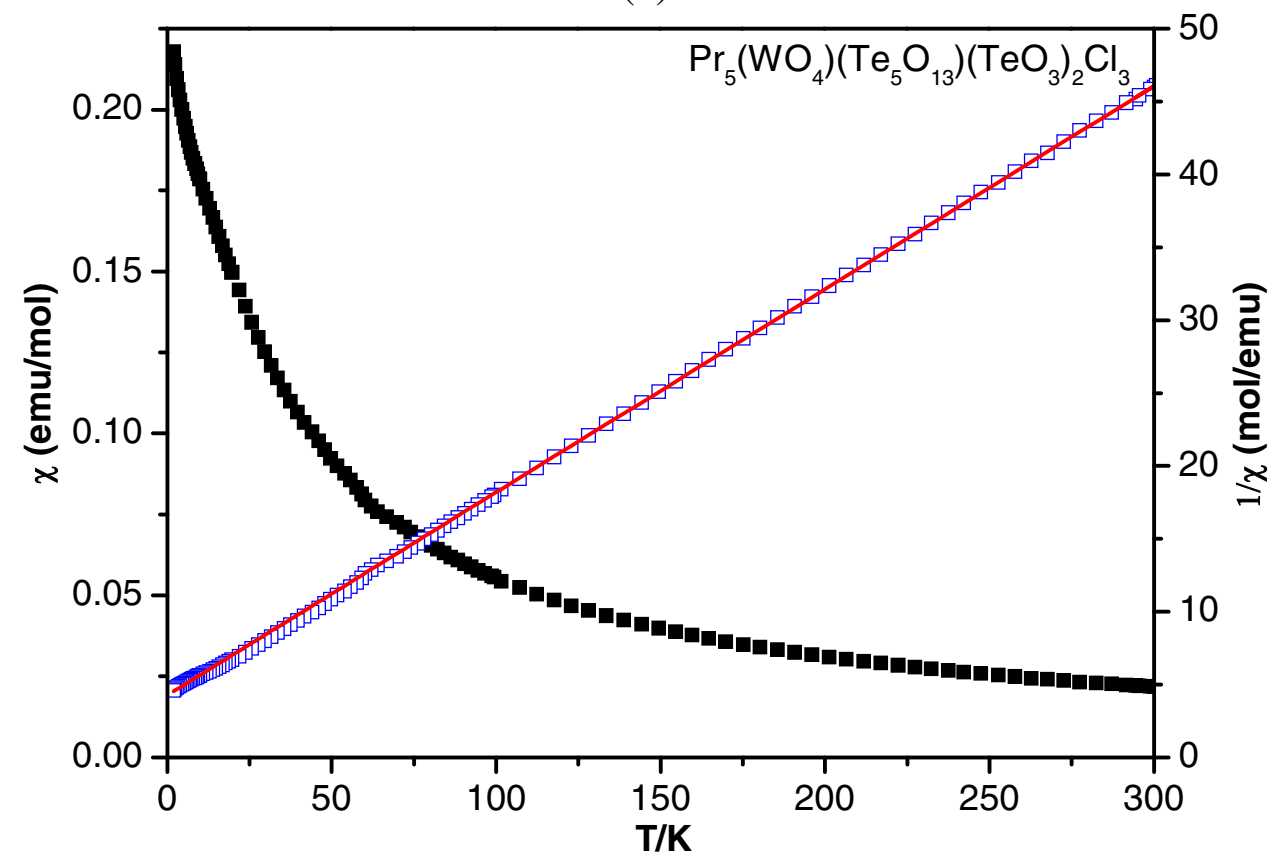

(e) 


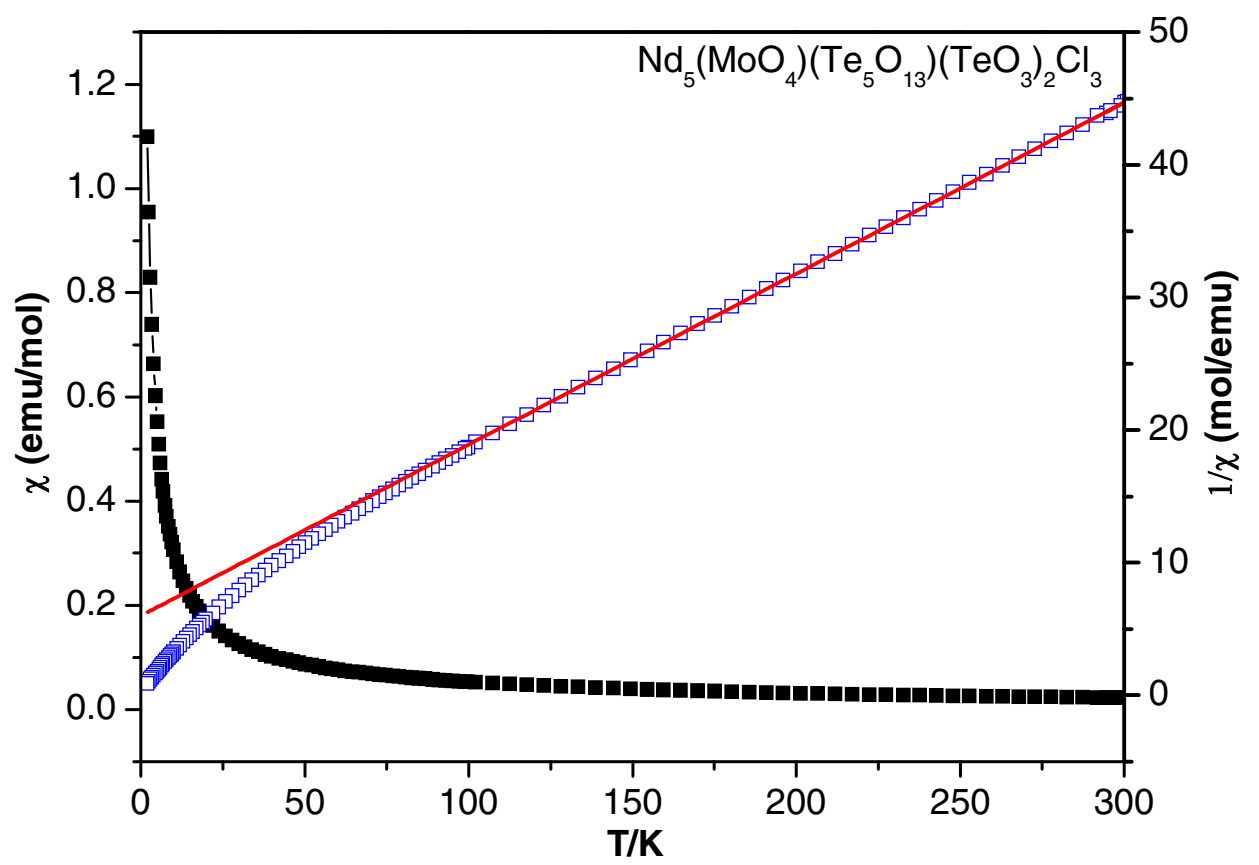

(f)

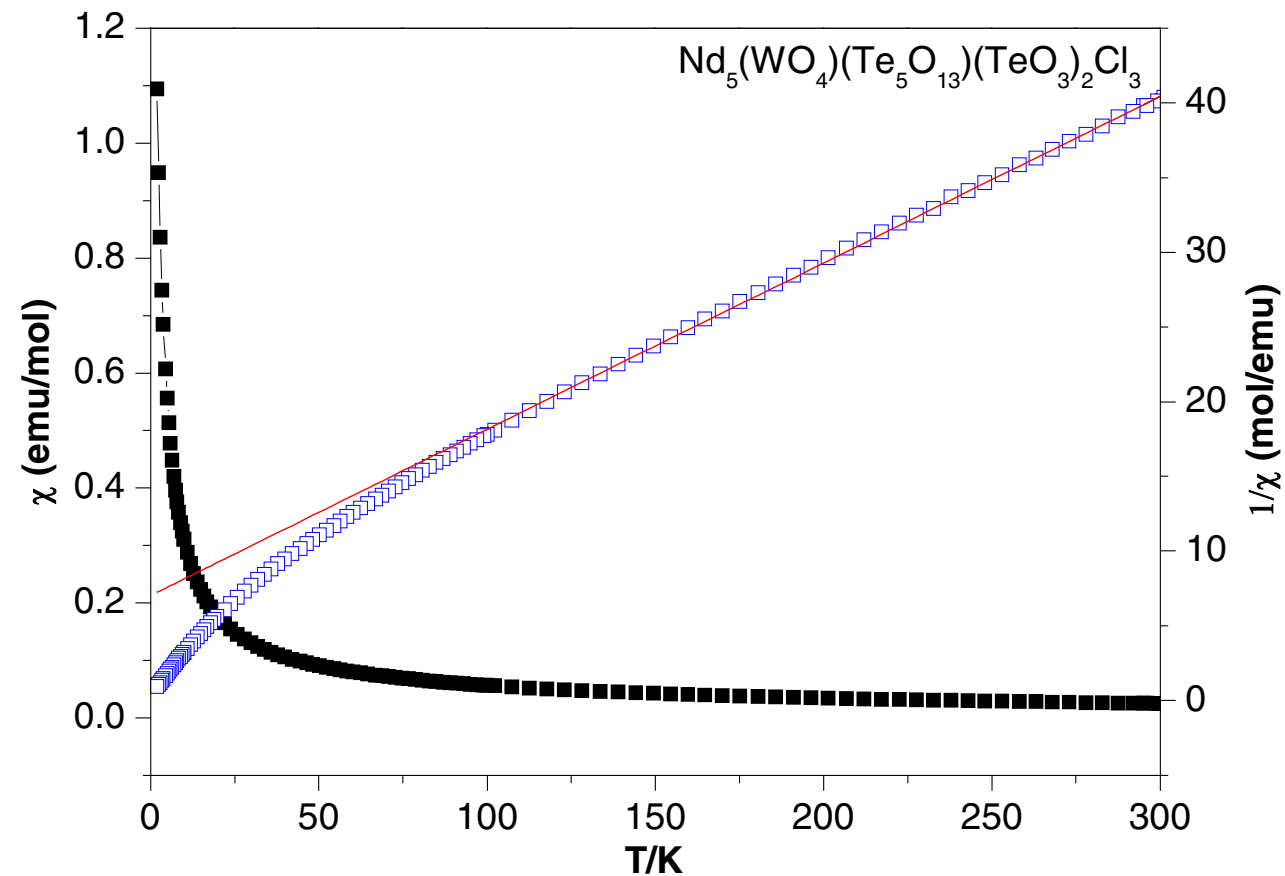

$(\mathrm{g})$

Figure S2. $\chi$ versus T and $1 / \chi$ versus $\mathrm{T}$ plots for $\operatorname{Pr}_{2}\left(\mathrm{MoO}_{4}\right)\left(\mathrm{Te}_{4} \mathrm{O}_{10}\right)(\mathrm{a}), \mathrm{Nd}_{2}\left(\mathrm{MoO}_{4}\right)\left(\mathrm{Te}_{4} \mathrm{O}_{10}\right)$
(b), $\quad \mathrm{Nd}_{2} \mathrm{~W}_{2} \mathrm{Te}_{2} \mathrm{O}_{13}$
(c),
$\operatorname{Pr}_{5}\left(\mathrm{MoO}_{4}\right)\left(\mathrm{Te}_{5} \mathrm{O}_{13}\right)\left(\mathrm{TeO}_{3}\right)_{2} \mathrm{Cl}_{3}$
(d),

$\mathrm{Pr}_{5}\left(\mathrm{WO}_{4}\right)\left(\mathrm{Te}_{5} \mathrm{O}_{13}\right)\left(\mathrm{TeO}_{3}\right)_{2} \mathrm{Cl}_{3}$

(e), $\quad \mathrm{Nd}_{5}\left(\mathrm{MoO}_{4}\right)\left(\mathrm{Te}_{5} \mathrm{O}_{13}\right)\left(\mathrm{TeO}_{3}\right)_{2} \mathrm{Cl}_{3}$

(f) and

$\mathrm{Nd}_{5}\left(\mathrm{WO}_{4}\right)\left(\mathrm{Te}_{5} \mathrm{O}_{13}\right)\left(\mathrm{TeO}_{3}\right)_{2} \mathrm{Cl}_{3}(\mathrm{~g})$. The red line corresponds to the linear fit of data according to the Curie-Weiss Law. 